\title{
Emerging Importance of Neuron-Satellite Glia Interactions within Trigeminal Ganglia in Craniofacial Pain
}

\author{
P.L. Durham* and F.G. Garrett
}

Center for Biomedical \& Life Sciences, Missouri State University, Springfield, MO 65806, USA

\begin{abstract}
Pain in the head and face, which can be very severe and debilitating, often involves activation of trigeminal ganglion nerves. The craniofacial symptoms can manifest as acute or transient conditions such as toothaches and headaches, or can transform into more chronic conditions such as migraine, rhinosinusitis, temporomandibular joint (TMJ) disorder, or trigeminal neuralgia. Traditionally, it is known that peripheral tissue injury or inflammation leads to excitation of trigeminal nerves that release inflammatory molecules in the periphery as well as facilitate transmission of nociceptive signals to the central nervous system. However, findings from recent studies have demonstrated that peripheral tissue injury or inflammation also leads to increased interactions between neuronal cell bodies and satellite glial cells within the trigeminal ganglion. These cell-to-cell interactions, which involve the transfer of key regulatory mediators via channels or gap junctions as well as paracrine signaling, are thought to play an important role in the induction and maintenance of peripheral sensitization of trigeminal nociceptors. The focus of this review will be on understanding the importance of the increased signaling between neuronal cell bodies and satellite glia cells in trigeminal ganglia to the development of persistent pain.
\end{abstract}

Keywords: Trigeminal, neuron, satellite glia, signaling, gap junctions - connexins, neuropathic, inflammation, acute, chronic, peripheral sensitization, comorbitity, migraine, TMJ, rhinosinusitus.

\section{TRIGEMINAL NERVES}

Pain in the head and face can be very severe and debilitating. In fact, the head and face represent some of the most common pain sites in the body [1-3]. These craniofacial symptoms can manifest as acute or transient conditions such as toothaches and headaches, or can transform into more chronic conditions such as migraine, rhinosinusitis, temporomandibular joint (TMJ) disorder or trigeminal neuralgia. While physiological pain is typically of short duration and facilitates an appropriate response to noxious stimuli, pathological pain is persistent, does not have longterm benefits to the organism and can lead to tissue damage and hence is maladaptive. Chronic diseases involving the head and face often involve activation of trigeminal ganglion nerves. The trigeminal nerve consists of three major branches that provide somatosensory innervation of distinct regions of the head, face, as well as nasal, sinus, and oral cavities [4]. Activation of a particular branch can be caused by peripheral tissue injury or compression of the nerve processes resulting in neuroinflammation or caused by tissue inflammation mediated by release of pro-inflammatory molecules at site of injury initiating a neurogenic inflammatory response. Following activation, trigeminal nerves release neuropeptides and other inflammatory molecules from peripheral terminals that initiate and maintain neurogenic inflammation, which is characterized by vasodilation, protein plasma leakage, mast cell activation,

*Address correspondence to this author at the Center for Biomedical \& Life Sciences , Missouri State University, Springfield, MO 65806;

Tel: (417) 836-4869; E-mail: pauldurham@ missouristate.edu and immune cell recruitment Fig. (1). The release of additional inflammatory molecules can lead to peripheral sensitization of trigeminal nociceptors. In addition, activation of trigeminal nerves by noxious stimuli will cause release of neuropeptides as well as glutamate from central terminating processes and cause excitation of second order neurons within the brainstem and spinal cord that are involved in transmission of nociceptive information leading to pain, central sensitization, and allodynia. More recently, peripheral activation is also thought to lead to excitation of the cell bodies of trigeminal neurons that increases glia-glia and neuron-glia signaling via gap junctions, as well as paracrine and autocrine mechanisms that together play a central role in regulating the excitability state of trigeminal neurons. Importantly, increased gap junction communication and release of ions and small signaling molecules within the ganglion is likely to also contribute to sensitization of nociceptive neurons. In this review, we will focus on recent studies that provide evidence of increased neuron-glia interactions during pathological conditions that are likely involved in the underlying pathology of persistent painful trigeminal nerve-mediated diseases.

The trigeminal or fifth cranial nerve is the largest and most complex of the twelve cranial nerves [4]. It has a large sensory (afferent) component and a small motor (efferent) component as it originates from the lateral border of the pons. The afferent fibers conduct the sensory information from various parts of the face, head, internal cranial structures, and TMJ. The efferent fibers send motor information to the muscles of mastication [4]. The trigeminal nerve is comprised of myelinated and unmyelinated nerve fibers that are responsible for conduction of nociceptive 


\section{Trigeminal Ganglion Neuron}

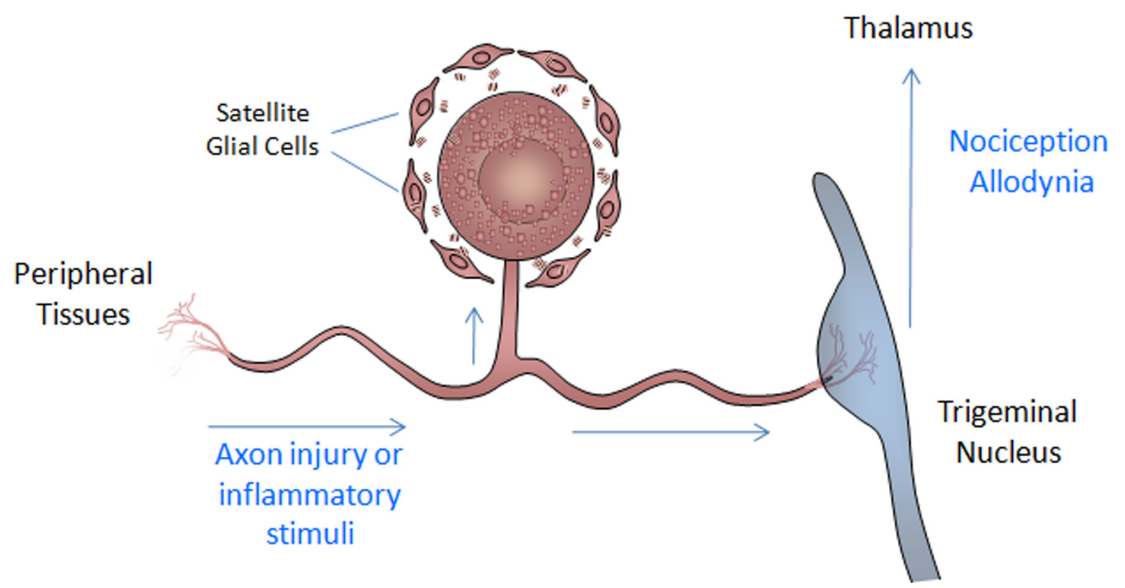

Fig. (1). Schematic of trigeminal ganglion neuron and associated satellite glial cells. In response to axon injury or inflammatory stimuli, the trigeminal neuron functions to transduce nociceptive signals from the periphery to the trigeminal nucleus resulting in central sensitization, allodynia, and pain. In addition, axon injury or inflammatory stimuli can cause increased neuron-glia interactions within the trigeminal ganglion that are involved in peripheral sensitization.

information from the peripheral tissues to the central nervous system [5]. Two types of myelinated nerve fibers that transmit nociceptive signals are seen in the trigeminal nerve: the thin myelinated $A \delta$ fibers that can function as lowthreshold mechanoreceptors, thermoreceptors, and nociceptors and the unmyelinated $\mathrm{C}$ fibers whose primary function is nociception [5]. The trigeminal nerve after exiting the skull forms a trigeminal ganglion where the cell bodies of the neurons reside. The trigeminal nerve then divides into the ophthalmic (V1) branch, the maxillary (V2) branch and the mandibular (V3) branch [4]. Activation of the $\mathrm{V} 1$ branch is implicated in the pain of migraine, cluster headache, sinus pathology, and trigeminal neuralgia, while activation of the V2 branch is associated with sinus pathology and trigeminal neuralgia, and TMJ disorders involve activation of the V3 branch.

\section{TRIGEMINAL GANGLION}

The trigeminal ganglion, also known as the gasserian or semilunar ganglion, is a large, flattened, semilunar shaped ganglion lying in the middle cranial fossa close to the cavernous sinus inside the skull, ensheathed between the two layers of the dura mater [4]. The ganglion contains the first order neurons of the sensory nerve fibers of the trigeminal nerve. The neurons in the trigeminal ganglion are pseudounipolar, where the cell body gives rise to a single axon from its axon hillock segment pole that then divides into a $\mathrm{T}$ or $\mathrm{Y}$ shape to give rise to a peripheral and a central projecting branch, a morphology similar to adult dorsal root ganglion neurons [6]. The peripheral fibers emerge from the convex side of the semilunar trigeminal ganglion as V1, V2, or V3 branches of the nerve, whereas the central processes project out of the concave side of the ganglion, enters the brainstem and terminates in the spinal trigeminal nucleus [4, 5].

The trigeminal ganglion is comprised of both neurons and glial cells. There are two distinct types of glia found in the trigeminal ganglion, the Schwann cells and the satellite glial cells [7]. Schwann cells are found in close proximity to the neuronal processes or axons where they function to produce myelin sheaths around axons that increase the conduction velocity of the nerve [8]. In contrast, neurons and satellite glial cells are arranged in discreet bands or clusters within each region of the adult rat trigeminal ganglion as seen in Fig. (2). Electron microscopic studies have shown the close proximity of the satellite glial cells with the neuronal cell bodies, with a distance of about $20 \mathrm{~nm}$ between the two cells [7]. Numerous flattened processes extend from satellite glial cells to the cell membrane of the neurons and are thought to facilitate communication between the neurons and the satellite glial cells via exchange of ions and small molecules. Similar to the cellular arrangement described for dorsal root ganglion, cell bodies of trigeminal ganglion neurons are completely surrounded by satellite glia that together form distinct, functional units [7]. Hence, satellite glial cells are thought to play an essential role influencing the activity of the neurons in response to peripheral injuries that lead to inflammation and pain [9]. The seminal work by Hanani and colleagues provided evidence that gap junction mediated signaling between satellite glial cells within the dorsal root ganglion is increased following nerve injury or inflammation [10-12]. However, the role of gap junctions within the trigeminal ganglion in promoting and sustaining trigeminal nerve mediated inflammation and pain is just beginning to be elucidated.

\section{Gap Junctions}

Gap junctions are specialized intercellular membrane channels that allow molecules less than $1 \mathrm{kDa}$ (i.e. secondary messengers, ions, and metabolic precursors) to pass directly from one cell to another $[13,14]$. Gap junctions, which are present in nearly all mammalian cell types [15, 16], are dynamic structures preprogrammed to be continuously biosynthesized and degraded in the cell that typically exhibit a short in vivo half-life between 1-5 hours [17-19]. Gap junctions do not typically function as a single unit but rather cluster together to form a tightly packed array known as a gap junction plaque. Importantly, evidence suggests that gap 


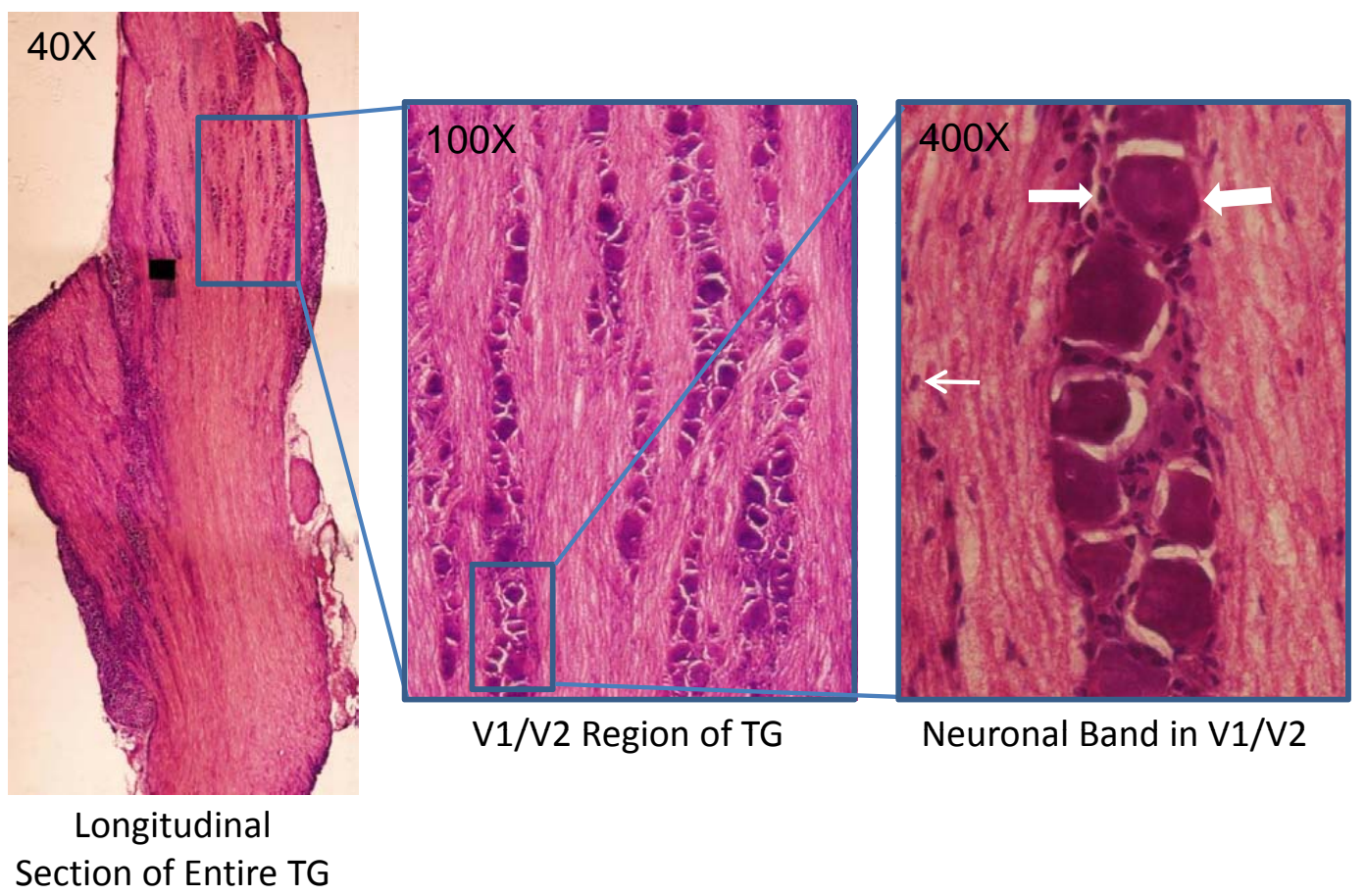

Fig. (2). $\mathrm{H} \& \mathrm{E}$ staining of trigeminal ganglion. An image of a longitudinal section of the entire ganglion is shown at $40 \mathrm{X}$ magnification in the left panel. As seen in the middle panel neurons and satellite glial cells are organized in bands or clusters within the ganglion. At higher magnification, individual satellite glial cells (thick arrows) are seen surrounding neuronal cell bodies, while individual Schwann cells (thin arrow) are found associated with neuronal fibers.

junction channels may not open and thus be functional until they cluster into plaques [20].

Gap junctions are composed of polytopic membrane proteins known as connexins (Cxs). Currently, the Cx family has 20 human members, ten of which have been identified in the nervous system [21, 22]. All members of the Cx family share a similar structure, where the proteins pass through the membrane four times creating two extracellular loops with the amino and carboxyl termini exposed to the cytoplasm [17]. Since Cx differ greatly in the size of the cytoplasmic loop region and the length of the carboxy terminal tail, each individual $\mathrm{Cx}$ is identified by their molecular weight $[15,23$, 24].

Six Cx proteins from one cell oligomerize into hexamers that are commonly referred to as either 'connexons' or 'hemichannels'. Thus, two adjacent hemichannels from opposing plasma membranes come together to form the gap junction channel [23]. In the nervous system, gap junctions have been shown to facilitate neuron-neuron, glia-glia, and neuron-glia communication $[25,26]$. Under normal neurological conditions, gap junction intercellular communication helps maintain a homeostatic environment by spatial buffering of important cellular ions such as $\mathrm{K}^{+}$, $\mathrm{Na}^{+}$and $\mathrm{Ca}^{2+}$ [27-30]. Consequently, changes in the expression of Cxs and hence, disruption of gap junction intracellular communication has been implicated in a number of CNS pathologies including: Alzheimer's disease, Parkinson's disease, epilepsy, and cortical spreading depression [25]. More recently, findings from studies on trigeminal ganglia designed to mimic nociceptive events that occur in human diseases have provided evidence of a key role of gap junction communication and increased neuron- glia interactions in modulating neuronal excitability and hence nociception.

\section{ROLE OF NEURON-SATELLITE GLIAL CELL IN- TERACTIONS IN MODELS OF NEUROPATHIC PAIN}

In the seminal work by Hanani and colleagues [11], axotomy of the infraorbital nerve resulted in significant changes in both neurons and satellite glial cells within the trigeminal ganglion. In response to nerve injury, there was increased coupling of satellite glial cells that temporally correlated with increased neuronal excitability as exemplified by an increase in the percentage of neurons firing spontaneously and a decrease in the threshold of activation. In an elegant study by Vit et al. [31], the investigators provide evidence of a fundamental role of gap junctions formed by $\mathrm{Cx} 43$ that couple trigeminal ganglion satellite glial cells in the development of spontaneous pain behavior. Specifically, their findings using in vivo RNAi demonstrated that increased $\mathrm{Cx} 43$ expression in satellite glial cells in response to chronic constriction of the infraorbital nerve was sufficient to induce pain. In a more recent study, the same investigators provided more definite evidence of the importance of glia in regulating the excitability state of trigeminal neurons under normal unstimulated conditions [32]. In support of their earlier findings, reducing $\mathrm{Cx} 43$ expression using RNAi in rats with a chronic constriction injury of the infraorbital nerve reduced pain-like behavior. However, somewhat surprisingly, suppressing Cx43 expression in non-injured animals increased pain-like behaviors to a level comparable to that of rats with chronic constriction injury. These results provide evidence that satellite glial cells play an important role in neuropathic pain 
states but also that disruption of their normal function can lead to pain.

In addition to gap junctions, other channels that are expressed by satellite glial cells are involved in regulating neuronal excitability. In particular, satellite glial cells were recently shown to express two ion channels, the inwardly rectifying potassium $\left(\mathrm{K}^{+}\right)$channel Kir4.1 as well as the smallconductance calcium activated potassium channel SK3, which function to maintain normal levels of extracellular $\mathrm{K}^{+}$ around neuronal cell bodies [31]. The control of $\mathrm{K}^{+}$levels is crucial for regulating the neuronal resting membrane potential and hence neuronal excitability. Importantly, increased neuronal excitability of primary sensory neurons is known to contribute to the development of persistent neuropathic pain by causing them to become spontaneously active or fire at a lower than normal threshold [11,33-35]. In a well-designed proof-of-concept study, compelling evidence of the importance of the Kir4.1 channel to regulate neuronal excitability and ultimately, the development of neuropathic pain was recently published [36]. Initially, the expression of Kir4.1 in satellite glial cells was found to decrease in response to chronic constriction injury of the infraorbital nerve. To determine the relevance of this finding to the development of neuropathic pain, expression of Kir4.1 in satellite glial cells was silenced using RNA interference. Decreased expression of Kir4.1 channel activity was found to cause spontaneous and evoked pain like behavior in free moving rats. Significantly, silencing Kir4.1 resulted in a reversible change in nociceptive threshold and nociceptiverelated behavioral changes, demonstrating that neuropathic pain can occur in response to changes in satellite glial cells to control the extracellular concentration of $\mathrm{K}^{+}$ions. It is likely that these findings will have important implications for regulation of extracellular glutamate levels as well since satellite glial cells express the glutamate transporter GLAST [31] whose ability to remove excess glutamate is modulated by Kir4.1 channel activity [37-39].

In a more recent study, a novel approach for reducing neuronal excitability and pain conditions using viral gene therapy was tested that involved increasing the level of the inhibitory transmitter gamma-aminobutyric acid (GABA) in trigeminal ganglia [40]. Injection of an adenoviral vector that contained the glutamic acid decarboxylase (GAD) gene directly into trigeminal ganglia caused increased expression of the GAD65 isoform and GABA synthesis mainly in satellite glial cells. Significantly, six days after injection, there was a statistically significant decrease in pain behavior in the orofacial formalin test, which is used as a model of inflammatory pain. Thus, transfection of glial cells in the ganglion with a GAD gene resulted in elevated levels of GABA that were sufficient to suppress acute pain behavior by acting on $\mathrm{GABA}_{\mathrm{A}}$ receptors on neuronal cell bodies. This finding is in agreement with the work of Naik and colleagues that provided evidence that direct injection of a $\mathrm{GABA}_{\mathrm{A}}$ receptor agonist into the dorsal root ganglion was antinociceptive in a model of peripheral nerve injury [41]. Taken together, data from these studies demonstrate the potential therapeutic benefit of directly increasing the amount of GABA in the ganglion, which functions to suppress neuronal activity that occurs in response to nerve damage and the development of peripheral and central sensitization.

\section{INCREASED NEURON-GLIA INTERACTIONS IN RESPONSE TO NOXIOUS STIMULI}

Neuronal-glial interactions are implicated in normal information processing, neuroprotection, and modulation of neuronal activity including rate of spontaneous firing and threshold of activation [7, 42-44]. Data from a recent study provided the first evidence of direct neuron to satellite glial cell communication within trigeminal ganglion in response to peripheral noxious stimuli [45]. Specifically, findings from dye coupling experiments demonstrated that direct neuronal-glial cell coupling via gap junctions was rapidly and markedly enhanced following activation of trigeminal neurons by the noxious stimulatory agent capsaicin. Stimulation of sensory neurons with capsaicin, which binds the TRPV1 receptor and causes excitation of nociceptive C fibers [46], is used as model of trigeminal nerve activation, a central event implicated in migraine pathology [47]. An interesting finding from the Thalakoti study [45] was that in response to capsaicin stimulation of only a few neurons within the V3 region of the ganglion, a time-dependent increase in the amount of the retrograde tracer dye True Blue in the cytosol of adjacent satellite glial cells and neighboring neurons was observed. Most of the dye, which was concentrated in neuronal cell bodies prior to stimulation, was localized in satellite glial cells surrounding neuronal cell bodies in the V3 region after 2 hours. It should be noted that although the dye was primarily localized in cell bodies of neurons under basal, unstimulated conditions, there did appear to be some minimal level of gap junction coupling between neurons and satellite glia, since some dye was found in a small percentage of satellite glial cells. However, increased gap junction coupling was only observed between neurons and satellite glial cells but not between neurons and Schwann cells. This finding is probably due to the fact that Schwann cells are physically not close enough to neuronal cell bodies in the trigeminal ganglion to facilitate direct signaling via gap junctions. Thus, based on these in vivo dyecoupling experiments, it appears that there is increased coupling and hence, increased communication between neurons and adjacent satellite glia in response to capsaicinmediated neuronal activation in the stimulated region of the ganglion.

What is the potential significance of increased signaling between neurons and glia within the trigeminal ganglion in response to nerve activation? While not fully understood, it has been shown that gap junctions formed between glial astrocytes allow for a syncytium-like organization that is responsible for the observed coordinated response and longdistance propagation of calcium waves in the CNS [48]. Thus, it is possible that satellite glial cells might perform a similar function to facilitate activation-dependent coupling that contributes to a coordinated inflammatory response within trigeminal ganglia. It is well established that gap junctions facilitate the movement of small molecules and ions such as calcium from one cell to another that allows them to be functionally coupled. Furthermore, capsaicin activation of trigeminal neurons has been reported to cause increased intracellular calcium levels [49]. In the Thalakoti study [45], levels of S100B, a member of the S100 family of calcium-binding proteins, were increased in neurons as well as satellite glial cells in the V3 region of the ganglion in 
response to capsaicin stimulation of V3 trigeminal nerves via injection into the TMJ capsule. Similar to the findings from the dye coupling experiment, there was no observed increase in S100B expression in Schwann cells. Functionally, S100 proteins are reported to regulate cell proliferation and differentiation as well as modulate the activity of key proteins such as signaling kinases, transcription factors, and cytoskeletal components in a calcium-dependent manner [50]. For example, S100B is reported to increase neuronal and glial levels of the transcription factor $\mathrm{NF}-\kappa \mathrm{B}$, which is known to stimulate expression of many genes involved in mediating inflammatory and nociceptive responses [51]. In addition to its involvement in intracellular signaling, S100B can be secreted from cells in response to inflammatory stimuli and function as a paracrine factor to cause changes in neighboring cells via activation of the cell surface receptor RAGE, which is expressed by neurons and glial cells [52]. Importantly, S100B has been reported to modulate neuronal function and its activation of RAGE has been shown to activate intracellular signaling pathways involving mitogenactivated protein (MAP) kinases [53]. The important contribution of MAP kinases in the induction and maintenance of peripheral sensitization and generation of persistent pain has recently been summarized [54].

A somewhat surprising finding from the Thalokoti study [45] was that activation of a few sensory neurons in the V3 region of the trigeminal ganglion resulted in intracellular changes in neighboring neuronal and glial cells within that same region but also in the V2 and V1 regions. In response to capsaicin injection into the TMJ capsule, S100B expression was observed not only in V3 neurons and satellite glial cells but was seen in both neurons and satellite cells in the V2 and V1 regions. A similar pattern was observed throughout the ganglion for the phosphorylated, active form of p38 MAP kinase in response to selective activation of neurons in the $\mathrm{V} 3$ region in response to tumor necrosis factor-alpha (TNF- $\alpha$ ) or nitric oxide (NO) donor and protons. Intraganglion communication involving crossdepolarization or cross-excitation was reported to occur between sensory neurons via release of diffusible molecules from the neuronal cell body in nodose ganglion [55]. This type of nonsynaptic communication, which has also been reported to occur in dorsal root ganglion, [56-58] would allow for a coordinated response to inflammatory stimuli. Activation of the p38 pathway in neuronal and glial cells has been reported to contribute to persistent inflammatory and neuropathic pain and its activation in nociceptive neurons may participate in generating pain hypersensitivity [59]. Further evidence for the involvement of p38 in pathological pain was demonstrated in a study in which inhibition of p38 was shown to alleviate inflammatory and neuropathic pain in animal models [60]. The role of increased p38 activation in neurons and satellite glial cells in trigeminal ganglia in response to inflammatory stimuli is not known. However, based on studies on dorsal root ganglion, increased p38 activity in neuronal and glial cells will likely contribute to peripheral sensitization of trigeminal nociceptive neurons and play an important role in the generation and maintenance of pathological pain [54].

Another protein likely involved in mediating crossexcitation of neurons and satellite glial cells within the trigeminal ganglion is the neuropeptide calcitonin gene- related peptide (CGRP), which is implicated in the underlying pathology of migraine and TMJ disorders [6164]. While most studies have focused on understanding the functions of CGRP released from peripheral or central terminals, there is evidence that sensory ganglion cell bodies are transiently depolarized and become more excitable by repetitive action potential activity in neighboring axons in the same ganglion $[57,58,65]$. Based on results from our study [45] and other studies on trigeminal ganglion [56, 66], CGRP released from the cell body of activated neurons would excite other neuronal cells and satellite glial cells via CGRP receptors expressed on these cells $[67,68]$. It is also likely that activation of trigeminal neurons would lead to CGRP release from neuronal processes that span across neuron-satellite glia bands within the ganglion. Interestingly, both neuronal cell bodies and neuronal processes express the vesicle docking protein SNAP-25 [69], which is involved in facilitating the stimulated release of CGRP from trigeminal neurons [70]. Thus, CGRP release from neuronal cell bodies or neuronal processes within the ganglion can function as an autocrine signal to increase synthesis and further release of CGRP [68].

In addition, CGRP release could function in a paracrine manner to excite other neurons as well as satellite glial cells. Toward this end, results from several in vitro studies provide evidence that CGRP activation of satellite glial cells stimulates increased release of several cytokines [45] and $\mathrm{NO}$ as well as upregulates the expression of inducible NO synthase (iNOS) via MAP kinase pathways [67]. Thus, release of cytokines, $\mathrm{NO}$, and other inflammatory molecules known to modulate neuronal function would generate a pathological inflammatory loop within the ganglion that sustains a hyperexcitable state of the neurons [71, 72]. In support of this notion, findings from a recent study provide evidence for the existence of a CGRP-cytokine inflammatory loop involving trigeminal ganglion neurons and satellite glial cells [73]. Data from this study demonstrate that satellite cells actively modulate trigeminal neuronal activity by amplifying and sustaining inflammatory processes within the ganglia. It is also likely that an inflammatory loop exists between CGRP and ATP within the trigeminal ganglion. Towards this end, CGRP has been shown to selectively increase membrane expression and activity of the ATP receptor $\mathrm{P} 2 \mathrm{X} 3$, leading to a sensitized state of trigeminal neurons [74]. Based on a study on dorsal root ganglia [65], ATP released from the soma of sensory neurons would activate $\mathrm{P} 2 \mathrm{X} 3$ receptors on trigeminal neurons and facilitate release of CGRP and other neurotransmitters that promote nociception. In addition, ATP released from the soma could activate $\mathrm{P} 2 \mathrm{X} 7$ receptors on satellite glial cells, leading to the release of TNF- $\alpha$, which in turn, potentiates $\mathrm{P} 2 \mathrm{X} 3$ receptor mediated responses and increases the excitability of nociceptive neurons. Furthermore, activation of satellite glial cells has recently been shown to lead to ATP release from those cells that would facilitate neuron-glia interactions [75]. This type of inflammatory loop has also been reported in response to pathological conditions in dorsal root ganglion where cross-depolarization and cross-excitation of neurons contributes to a hyperexcitability state characteristic of injured dorsal root ganglion nerves [55]. Collectively, these results support a model by which activation of neurons and satellite glial cells in one region of the ganglion initiate an 
inflammatory cascade involving other neurons as well as satellite glial cells, leading to increased intraganglion and neuron-glia communication in sensory ganglia. Taken together, these data may provide a cellular basis for the significant comorbidity associated with diseases involving trigeminal nerves $[76,77]$.

\section{MODEL OF COMORBID DISEASES - SINUS PA- THOLOGY AND MIGRAINE}

Interestingly, patients with migraine headache often cite sinus pain and pressure as a cause or trigger of their headaches [76, 78, 79]. Is it possible that cross-excitation (propagation of inflammatory signals) within the ganglion lowers the activation threshold of neurons involved in migraine pathology and thus helps explain the commonly reported symptoms of comorbid conditions associated with migraine? A recent in vivo study was designed to directly test the hypothesis that the unique cellular morphology of the trigeminal ganglion allows cell to cell signaling within the ganglion that mediates activation of other branches leading to comorbidity as reported for migraine and allergic rhinitis and acute sinusitis [80]. In the study by Damodaram et al. [81], injection of TNF- $\alpha$, a cytokine whose levels are elevated in nasal secretions during allergic rhinitis and acute sinusitis [82-85], in a facial region (whisker pad) that is innervated by $\mathrm{V} 2$ neurons lowered the activation threshold to capsaicin in V1 neurons. Sensitization and activation of trigeminal neurons originating in the $\mathrm{V} 1$ region of the ganglion that provide sensory innervation of the meningeal blood vessels are thought to be involved in the pathology of migraine [86]. Importantly, it was shown that TNF- $\alpha$ or capsaicin treatment alone was not sufficient to cause cellular changes in V1 neurons. However, the combination of both chemical stimuli at subthreshold levels was sufficient to facilitate gap junction communication between trigeminal neurons and satellite glial cells and increase active p38 levels in both cell types within the $\mathrm{V} 1$ and $\mathrm{V} 2$ regions. In agreement with our previous study [45], the increase in gap junction signaling and p38 levels was observed not only in the $\mathrm{V} 1$ region but also in the $\mathrm{V} 2$ region of the ganglion. Based on results from these studies, it appears that this type of intraganglion signaling may be a normal cellular response to peripheral stimulation of trigeminal neurons. While increased expression of signaling molecules across different regions of the trigeminal ganglion is likely to involve neuron to glia signaling via gap junctions and paracrine signaling as discussed above, other mechanisms may also play a central role. Interestingly, retrograde labeling of neurons whose cell bodies were thought to be localized exclusively in one branch of the ganglion revealed an overlapping distribution of neuronal cell bodies in the V1 and V2 regions. As an example, injection of the tracer dye True Blue in the whisker pads, which is thought to be innervated solely by V2 neurons, labeled neuronal cell bodies organized in bands in the V2 region but also labeled neuron cell bodies in the V1 region. Thus, the observed overlapping distribution of neuronal cells in bands within the $\mathrm{V} 1$ and $\mathrm{V} 2$ regions would allow for a coordinated response to inflammatory stimuli that may account for why cellular changes in one branch leads to coordinated changes in the other region of the trigeminal ganglion. In addition, it should be noted that comorbidity is likely to not only be caused by changes within the ganglia but will involve convergence of primary afferents at the level of the trigeminal nucleus caudalis and central sensitization of secondary neurons involved in pain transmission [87, 88]. Taken together, these findings provide evidence that may help, at least in part, to explain how rhinosinusitis or acute sinusitis may act as a trigger of migraine as well as provide a possible explanation for the significant comorbidity seen between migraine and rhino-sinus diseases.

In the same study, increased neuron-satellite glial cell signaling via gap junctions was greatly increased in both V1 and $\mathrm{V} 2$ regions of the trigeminal ganglion in response to TNF- $\alpha$ and then capsaicin treatment [81]. Based on data from this study, gap junctions that allowed for direct dye coupling of neurons and satellite glial cells in trigeminal ganglion appear to be primarily composed of $\mathrm{Cx} 26$ proteins. This is the first report of $\mathrm{Cx} 26$ being expressed by neurons and satellite glial cells in the trigeminal ganglion. While Cx26 expression had previously been reported in CNS neurons and astrocytes [17, 21, 24], this was the first demonstration of Cx26 expression in satellite glial cells. A significant finding from this study was that the drug tonabersat, a member of a family of novel benzoylaminobenz compounds, was shown to greatly diminish dye coupling between trigeminal neurons and satellite glial cells and decrease the levels of Cx26 in both cell types [81]. In addition, tonabersat treatment was shown to block the stimulatory effect of TNF- $\alpha$ and capsaicin treatment on active p38 levels. It is not known if the inhibitory effect of tonabersat on p38 levels is mediated by a direct mechanism or an indirect mechanism by reducing signaling via $\mathrm{Cx} 26$ gap junctions. Results from this study provided evidence to suggest that tonabersat can inhibit key cellular events that contribute to peripheral sensitization in trigeminal ganglia, and therefore should be effective as a therapeutic for migraine, allergic rhinitis, TMJ disorders, and possibly the neuropathic pain associated with trigeminal neuralgia.

\section{NEURON-GLIA INTERACTIONS IN A MODEL OF TMJ PATHOLOGY}

A novel in vivo model of acute TMJ inflammation was used to investigate the cell signaling pathways activated in trigeminal ganglion neurons and glia in response to nerve stimulation with NO donor and protons [89]. The decision to study the effects of NO-protons was based on the fact that elevated levels of these molecules in human TMJ capsules are implicated in joint inflammation and pain [90, 91]. While NO can exert direct stimulatory effects on the neurons, the stimulatory effect of protons on trigeminal neurons likely involve activation of the proton-sensitive ion channels TRPV1 or ASIC3, which are known to be expressed by trigeminal nociceptors [45, 92-95]. In the study by Freeman et al., [89] injection of an NO donor diluted in HBS at $\mathrm{pH}$ 5.5 into both joint capsules resulted in temporal and spatial changes in the expression of MAP kinases and MAP kinase phosphatases (MKPs)in both neurons and satellite glial cells in all regions of the trigeminal ganglion. Specifically, the phosphorylated, active forms of p38 and ERK, but not the MAP kinase JNK, were significantly elevated in the cytosol and nucleus of neurons and satellite glial cells within $15 \mathrm{~min}$ of NO-proton stimulation of V3 trigeminal neurons. Active levels of these proteins remained elevated at the $2 \mathrm{~h}$ time point when compared to control levels. Although cytosolic 
and nuclear staining for p38 remained greater than basal levels even $24 \mathrm{~h}$ after injection, the level of ERK staining in the cytosol and nucleus had returned to control levels. The rapid increase in these MAP kinases may be involved in posttranslational events mediated by phosphorylation of ion channels and receptors that are reported to occur within minutes and lead to peripheral sensitization of nociceptors [59]. The prolonged increase in p38 in neurons and satellite glia would regulate pro-inflammatory and pro-nociceptive genes responsible for maintaining peripheral sensitization as well as an enhanced pain state [54]. In other models of tissue injury and inflammation, sensitizing agents such as NO and protons that are released from inflammatory cells and nerve terminals have been reported to cause activation of multiple protein kinases involved in neuronal signal transduction including ERK and p38 MAP kinases [59].

Increased expression of p38 and ERK in the nucleus of trigeminal ganglion neurons is likely to cause increased expression of genes such as CGRP and iNOS that are known to be MAP kinase-responsive [96, 97] and that are implicated in the underlying pathology of TMJ disorders [63, 64]. Similarly, increased expression of p38 and ERK in satellite glial cells would be expected to lead to induction of pro-inflammatory genes such as cytokines and interleukins reported to be regulated by these MAP kinases and expressed by satellite glial cells [98, 99]. Of clinical relevance, elevated levels of interleukin $1-\beta$, interleukin 6 , and TNF- $\alpha$ have been reported in synovial fluid obtained from TMJ patients $[100,101]$. In another study, chemical activation of trigeminal neurons that provide sensory innervation to the whisker pads caused increased expression of interleukin 1- $\beta$, which mediates inflammation and hyperalgesia and is known to be MAP kinase-responsive, in satellite glial cells [102]. Thus, it is now thought that activation of satellite glial cells mediate enhanced excitability of nociceptive trigeminal ganglion neurons following peripheral inflammation [44, 102].

An important point to consider is how MAP kinase levels in neurons and satellite glial cells return to basal levels following trigeminal nerve activation since the magnitude and duration of MAP kinase stimulation is a crucial determinant of the biological outcome. In mammalian cells, the active levels of MAP kinases are negatively regulated by the activity of MKPs. In the study by Freeman et al. [89], NO-proton stimulation of sensory afferents in the TMJ caused spatial and temporal changes in the levels of several MKPs in the neurons and satellite glial cells in all regions of the trigeminal ganglion. Interestingly, MKP-1 expression was readily detectable in satellite glial cells in all regions of the ganglion under basal conditions, in contrast to levels in trigeminal neurons that were barely detectable. It is possible that this level of MKP-1 in satellite glial cells is important for regulating the expression and possibly release of cytokines, interleukins, as well as other inflammatory molecules. In support of this notion, MKP-1 regulates the output of cytokines involved in an inflammatory response by limiting the strength and duration of p 38 and JNK activation [103]. While only MKP-1 levels were elevated in satellite glial cells under basal conditions, the expression of MKP-1, MKP-2, and MKP-3 were all significantly elevated in both neurons and glial cells in all regions of the ganglion $2 \mathrm{~h}$ after NO-proton stimulation. However, only the levels of MKP-2 and MKP-3 remained significantly elevated $24 \mathrm{~h}$ after injection of inflammatory stimuli. While it is likely that induction of these three MKPs play a role in modulating the levels of p38 and ERK in neuronal and satellite glial cells, it is not known which MKP is primarily responsible for decreasing p38 or ERK levels. In addition, it is possible that other members of the MKP family may also be involved in regulating p38 and ERK, and hence, activation and excitation of trigeminal neuronal and satellite glial cells. Thus, it appears that NO-proton stimulation of trigeminal neurons leads to an initial increase in the active levels of $\mathrm{p} 38$ and ERK in neurons and satellite glial cells as well as a prolonged induction of several MKPs. The increased levels of these MKPs, and possibly others, would lower elevated p38 and ERK levels to basal levels and be expected to suppress peripheral sensitization in the trigeminal ganglion nerves, inhibit inflammation in the TMJ, and prevent transmission of painful stimuli.

\section{CHANGES IN CONNEXIN EXPRESSION IN RE- SPONSE TO ACUTE OR CHRONIC NOXIOUS STIMULI}

Data from previous studies had provided evidence of increased communication via gap junctions between trigeminal ganglion neurons and the surrounding satellite glia in response to acute inflammatory stimuli $[45,81]$ and between satellite glia in a neuropathic pain model [31, 32, 36]. While neuron-satellite glial cell interactions were shown to involve increased expression of $\mathrm{Cx} 26$, satellite gliasatellite glia signaling through gap junctions was mediated by $\mathrm{Cx} 43$. In a recently published study, temporal and spatial changes in $\mathrm{Cx}$ expression in both trigeminal ganglion neurons and satellite glial cells under basal conditions as well as in a chronic and an acute model of TMJ inflammation were investigated [104]. Under basal conditions, mRNA and protein for Cxs 26, 36, 40, and 43 were present in trigeminal ganglia isolated from untreated animals. A significant finding from this study was that the duration of $\mathrm{Cx}$ expression in trigeminal ganglion neurons and satellite glial cells temporally correlated with the type of inflammatory stimulus injected into the TMJ capsule. For example, injection of complete Freund's adjuvant (CFA), which is known to cause a strong and long-lasting inflammatory response in the joint [105-108], into the TMJ capsule resulted in a sustained increase in Cx26 expression in neurons and satellite glial cells as well as sustained expression of $\mathrm{Cx} 36$ and $\mathrm{Cx} 40$ in neurons when compared to basal levels. It is most probable that a sustained increase in Cx26 expression in both neurons and satellite glial cells facilitates gap junction communication by allowing molecules such as cAMP, glutamate, as well as $\mathrm{K}^{+}, \mathrm{Na}^{+}$, and $\mathrm{Ca}^{2+}$ ions to pass directly between these cells in the ganglion for a prolonged period of time. Importantly, increased neuronal-glial cell signaling via gap junctions has been documented in CNS inflammatory diseases and is considered an important factor in the underlying pathology by increasing neuronal excitability [109]. Thus, similarly increased neuron-satellite glia coupling via gap junctions in trigeminal ganglia would facilitate increased neuronal excitability and lower the activation threshold to inflammatory stimuli. In addition, given that $\mathrm{Cx} 36$ and $\mathrm{Cx} 40$ expression was increased primarily in neurons and the fact 
that these Cxs are known to form hemichannels [110, 111], their increased expression in response to inflammatory stimuli could result in enhanced autocrine and paracrine signaling within the ganglion that also would increase the excitability state of the neurons and satellite glial cells. In support of this notion, hemichannels have been found to act through paracrine signaling by releasing molecules such as ATP, NAD+, glutamate, and prostaglandins, which can modulate neuronal and glial cell activity [23, 112-116].

In contrast to the sustained increase in $\mathrm{Cx}$ expression observed in response to CFA, injection of capsaicin caused a transient elevation in Cxs 26, 36, and 40 levels [104]. Elevated levels of those three Cxs were observed within 15 minutes after capsaicin injection and remained elevated for at least 2 hours post injection. However, by 24 hours, levels of Cxs 26, 36, and 40 had returned to basal levels. Despite the temporal differences in response to CFA and capsaicin injections observed in our study, the spatial expression of each $\mathrm{Cx}$ was similar. For example, while Cx26 expression was markedly increased in both neurons and satellite glial cells in response to capsaicin, increased Cx36 and Cx40 expression was primarily localized to neuronal cell bodies as seen following CFA injections. Based on previous studies $[45,81]$, the changes in Cx expression likely facilitate direct communication of neurons and satellite glial cells via gap junctions and possibly enhanced autocrine and paracrine signaling in the ganglion that contributes to increased neuronal and glial excitability. Of relevance to TMJ pathology, an increase in nociceptor excitability is characteristic of both peripheral sensitization, which occurs in response to an acute inflammatory stimulus, and priming, which is a condition involving long-term changes in the excitability state of sensory nociceptive neurons [117]. Peripheral sensitization, which can last from minutes to hours, is characterized by increased neuronal excitability and a lowering of the threshold stimulus for increasing gene expression, ion channel activities, and release of inflammatory molecules [42]. More recently, a primed state of nociceptors has been proposed in which significantly lower concentrations of inflammatory mediators are required to elicit a heightened state of pain (hyperalgesia) that can persist for several weeks [117]. Thus, given the importance of glial cells in the regulation of neuronal excitability and activation thresholds $[7,102,118]$, it is possible that the transient increased $\mathrm{Cx}$ expression observed in response to capsaicin contributes to peripheral sensitization, while the more stable expression of Cxs in response to CFA is involved in the generation and/or maintenance of the primed state. Furthermore, it is probable that the sustained increase in $\mathrm{Cx}$ expression may play a central role in the transition from acute episodic pain in the TMJ to a more chronic pain state.

A particularly interesting finding was that expression of Cx43 was not increased in response to CFA or capsaicin injection into the TMJ, although $\mathrm{Cx} 43 \mathrm{mRNA}$ was detected in trigeminal ganglia from untreated animals and a low level of Cx43 immunoreactivity was detected in satellite glial cells [104]. These data are in contrast to the increased expression of $\mathrm{Cx} 43$ reported in trigeminal ganglion satellite glia in response to trigeminal nerve injury $[31,32]$. Thus, it appears the Cx43 expression in satellite glial cells is differentially regulated based on the type of stimulus. Furthermore, this fundamental difference in the response of satellite glial cells to activation of trigeminal neurons due to injury or noxious stimuli is likely to have important therapeutic implications.

\section{SUMMARY}

In conclusion, findings from animal studies designed to mimic some aspects of human disease have provided evidence of increased neuronal-glial cell interactions in trigeminal ganglion in response to peripheral nociceptor activation caused by tissue injury or inflammation. Results from those studies support an emerging central role of neuron-glia interactions within trigeminal ganglia in peripheral sensitization as well as induction and maintenance of persistent pain states. Given the prevalence and comorbidity associated with migraine, rhinosinusitis, and TMJ disorders as well as neuropathic pain, a better understanding of the normal function of satellite glial cells and ways to modulate their activity under pathological conditions has considerable health implications. While there is much evidence to support the important role of increased neuron-glia interactions to the development of hyperalgesia as well as chronic pain within the CNS, the significance of neuron-satellite glial cell interactions within trigeminal ganglia is only now being elucidated. In particular, it is becoming clear that satellite glial cells function in a capacity beyond merely supporting neuronal cell bodies but play an essential function in neuronal homeostasis by regulating the extracellular levels of $\mathrm{K}^{+}$ions and glutamate and thus, the excitability state of trigeminal nociceptors. Furthermore, we propose that neuronal-glial cell communication via gap junctions and paracrine signaling are also involved in the development of peripheral sensitization within the trigeminal ganglion, and therefore play an important role in the underlying pathology of diseases involving trigeminal nerve activation.

\section{REFERENCES}

[1] Sessle B. The neurobiology of facial and dental pain: Present knowledge, future directions. J Dent Res 1987; 66: 962-981.

[2] Lipton JA, Ship JA, Larach-Robinson D. Estimated prevalence and distribution of reported orofacial pain in the united states. J Am Dent Assoc 1993; 124: 115-121.

[3] Carlsson G, LeResche L. Epidemiology of temporomandibular disorders. In: Sessle, Bryant PS, Dionne, RA (Eds). Temporomandibular disorders and related pain conditions. Progress in pain research and management, IASP Press, Seattle, 1995, pp. 211-226.

[4] Shankland W. The trigeminal nerve. Part i: An over-view. Cranio 2000; 18: 238-248

[5] Lazarov N. Comparative analysis of the chemical neuroanatomy of the mammalian trigeminal ganglion and mesencephalic trigeminal nucleus. Prog Neurobiol 2002; 66: 19-59.

[6] Devor M. Unexplained peculiarities of the dorsal root ganglion. Pain 1999; Suppl 6: S27-35.

[7] Hanani M. Satellite glial cells in sensory ganglia: From form to function. Brain Res Rev 2005; 48: 457-476.

[8] Garbay B, Heape A, Sarqueil F, et al. Myelin synthesis in the peripheral nervous system. Prog Neurobiol 2000; 61: 267-304.

[9] Huang T, Belzer V, Hanani M. Gap junctions in dorsal root ganglia: Possible contribution to visceral pain. Eur J Pain 2010; 49: e1-11.

[10] Hanani M, Huang $\mathrm{T}$, Cherkas $\mathrm{P}$, et al. Glial cell plasticity in sensory ganglia induced by nerve damage. Neuroscience 2002; 114: 279-283.

[11] Cherkas P, Huang T, Pannicke T, et al. The effects of axotomy on neurons and satellite glial cells in mouse trigeminal ganglion. Pain 2004; 110: 290-298. 
[12] Dublin P, Hanani M. Satellite glial cells in sensory ganglia: Their possible contribution to inflammatory pain. Brain Behav Immun 2007; 21: 592-598.

[13] Goldberg GS, Lampe PD, Nicholson BJ. Selective transfer of endogenous metabolites through gap junctions composed of different connexins. Nat Cell Biol 1999; 1: 457-459.

[14] Goldberg G, Moreno A, Lampe P. Gap junctions between cells expressing connexin 43 or 32 show inverse permselectivity to adenosine and atp. J Bio Chem 2002; 277: 36725-36730.

[15] White T, Bruzzone R. Multiple connexin proteins in single intercellular channels: Connexin compatibility and functional consequences. J Bioenerg Biomembr 1996; 28: 339-350.

[16] Beyer E, Davis L, Saffitz J, et al. Cardiac intercellular communication: Consequences of connexin distribution and diversity. Braz J Med Biol Res 1995; 4: 415-425.

[17] Laird D. Life cycle of connexins in health and disease. Biochem $\mathbf{J}$ 2006; 394: 527-543.

[18] Fallon R, Goodenough D. Five-hour half-life of mouse liver gapjunction protein. J Cell Biol 1981; 90: 521-526.

[19] Beardslee M, Laing J, Beyer E, et al. Rapid turnover of connexin43 in the adult rat heart. Circ Res 1998; 83: 629-635.

[20] Bukauskas F, Jordan K, Bukauskiene A, et al. Clustering of connexin 43-enhanced green flourescent protein gap junction channels and functional coupling in living cells. Proc Natl Acad Sci USA 2000; 97: 2556-2561.

[21] Nagy J, Dudek F, Rash J. Update on connexins and gap junctions in neurons and glia in the mammalian nervous system. Brain Res Rev 2004; 47: 191-215.

[22] Ebihara L. New roles for connexons. News Physiol Sci 2003; 18 : 100-103.

[23] Goodenough D, Goliger J, Paul D. Connexins, connexons, and intercellular communication. Annu Rev Biochem 1996; 65: 475502.

[24] Saez J, Berthoud V, Branes M, et al. Plasma membrane channels formed by connexins: Their regulation and function. Physiol Rev 2003; 83: 1359-1400

[25] Rouach N, Avignone E, Meme W, et al. Gap junctions and connexin expression in the normal and pathological central nervous system. Biol Cell 2002; 94: 457-475.

[26] Alvarez-Maubecin V, Garcia-Hernandez F, Williams J, et al. Functional coupling between neurons and glia. J Neurosci 2000; 20: 4091-4098.

[27] Venance L, Prémont J, Glowinski J, et al. Gap junctional communication and pharmacological heterogeneity in astrocytes cultured from the rat striatum. J Physiol 1998; 510: 429-440.

[28] Mobbs P, Brew H, Attwell D. A quantitative analysis of glial cell coupling in the retina of the axolotl (ambystoma mexicanum). Brain Res 1998; 460: 235-245.

[29] Holthoff K, Witte O. Directed spatial potassium redistribution in rat neocortex. Glia 2000; 29: 288-292.

[30] Rose C, Ransom B. Gap junctions equalize intracellular na+ concentration in astrocytes. Glia 1997; 20: 299-307.

[31] Vit J, Jasmin L, Bhargava A, et al. Satellite glial cells in the trigeminal ganglion as a determinant of orofacial neuropathic pain. Neuron Glia Biol 2006; 2: 247-257.

[32] Ohara P, Vit J, Bhargava A, et al. Evidence for a role of connexin 43 in trigeminal pain using rna interference in vivo. J Neurophys 2008; 100: 3064-3073.

[33] Amir R, Devor M. Extra spike formation in sensory neurons and the disruption of afferent spike patterning. Biophys J 2003; 84: 2700-2708.

[34] Amir R, Devor M. Electrical excitability of the soma of sensory neurons is required for spike invasion of the soma, but not for through-conduction. Biophys J 2003; 84: 2181-2191.

[35] Robinson P, Boissonade F, Loescher A, et al. Peripheral mechanisms for the initiation of pain following trigeminal nerve injury. J Orofac Pain 2004; 18: 287-292.

[36] Vit JP, Ohara PT, Bhargava A, et al. Silencing the kir4.1 potassium channel subunit in satellite glial cells of the rat trigeminal ganglion results in pain-like behavior in the absence of nerve injury. $\mathbf{J}$ Neurosci 2008; 28: 4161-4171.

[37] Kucheryavykh Y, Kucheryavykh L, Nichols C, et al. Downregulation of kir4.1 inward rectifying potassium channel subunits by rnai impairs potassium transfer and glutamate uptake by cultured cortical astrocytes. Glia 2007; $55: 274-281$.
[38] Djukic B, Casper K, Philpot B, et al. Conditional knock-out of kir4.1 leads to glial membrane depolarization, inhibition of potassium and glutamate uptake, and enhanced short-term synaptic potentiation. J Neurosci 2007; 27: 11354-11365.

[39] Olsen M, Sontheimer H. Functional implications for kir4.1 channels in glial biology: From k+ buffering to cell differentiation. J Neurochem 2008; 107: 589-601.

[40] Vit J, Ohara P, Sundberg C, et al. Adenovector gad65 gene delivery into the rat trigeminal ganglion produces orofacial analgesia. Mol Pain 2009; 5: 42.

[41] Naik A, Pathirathna S, Jevtovic-Todorovic V. Gabaa receptor modulation in dorsal root ganglia in vivo affects chronic pain after nerve injury. Neuroscience 2008; 154: 1539-1553.

[42] Dodick D, Silberstein S. Central sensitization theory of migraine: Clinical implications. Headache 2006; 46: S182-191.

[43] Watkins L, Milligan E. Maier S. Glial activation: A driving force for pathological pain. Trends Neurosci 2001; 24: 450-455.

[44] Takeda M, Takahashi M, Matsumoto S. Contribution of the activation of satellite glia in sensory ganglia to pathological pain Neurosci Biobehav Rev 2009; 33: 784-792.

[45] Thalakoti S, Patil V, Damodaram S, et al. Neuron-glia signaling in trigeminal ganglion: Implications for migraine pathology. Headache 2007; 47: 1008-1023.

[46] Caterina M, Schumacher M, Tominaga M, et al. The capsaicin receptor: A heat-activated ion channel in the pain pathway. Nature 1997; 389: 816-824.

[47] Pietrobon D, Striessnig J. Neurobiology of migraine. Nat Rev Neurosci 2003; 4: 386-398.

[48] Blomstrand F, Aberg N, Eriksson P, et al. Extent of intercellular calcium wave propagation is related to gap junction permeability and level of connexin-43 expression in astrocytes in primary cultures from four brain regions. Neuroscience 1999; 92: 255-265.

[49] Garcia-Hirschfeld J, Lopez-Briones L, Belmonte C, et al. Intracellular free calcium responses to protons and capsaicin in cultured trigeminal neurons. Neuroscience 1995; 67: 235-243.

[50] Donato R. S100: A multigenic family of calcium-modulated proteins of the ef-hand type with intracellular and extracellular functional roles. Int J Biochem Cell Biol 2001; 33: 637-668 Review.

[51] Liu L, Li Y, Van Eldik LJ, et al. S100b-induced microglial and neuronal il-1 expression is mediated by cell type-specific transcription factors. J Neurochem 2005; 92: 546-553.

[52] Lue LF, Walker DG, Brachova L, et al. Involvement of microglial receptor for advanced glycation endproducts (rage) in alzheimer's disease: Identification of a cellular activation mechanism. Exp Neurol 2001; 171: 29-45.

[53] Marenholz I, Heizmann CW, Fritz G. S100 proteins in mouse and man: From evolution to function and pathology (including an update of the nomenclature). Biochem Biophys Res Commun 2004; 322: 1111-1122.

[54] Cheng J, Ji R. Intracellular signaling in primary sensory neurons and persistent pain. Neurochem Res 2008; 33: 1970-1978.

[55] Oh EJ, Weinreich D. Chemical communication between vagal afferent somata in nodose ganglia of the rat and the guinea pig in vitro. J Neurophysiol 2002; 87: 2801-2807.

[56] Ulrich-Lai YM, Flores CM, Harding-Rose CA, et al. Capsaicinevoked release of immunoreactive calcitonin gene-related peptide from rat trigeminal ganglion: Evidence for intraganglionic neurotransmission. Pain 2001 91: 219-226.

[57] Amir R, Devor M. Chemically mediated cross-excitation in rat dorsal root ganglia. J Neurosci 1996; 16: 4733-4741.

[58] Amir R, Devor M. Functional cross-excitation between afferent aand c-neurons in dorsal root ganglia. Neuroscience 2000; 95: 189195.

[59] Ji RR. Peripheral and central mechanisms of inflammatory pain, with emphasis on map kinases. Curr Drug Targets Inflamm Allergy 2004; 3: 299-303.

[60] Ji R. Mitogen-activated protein kinases as potential targets for pain killers. Curr Opin Investig Drugs 2004; 5: 71-75.

[61] Hargreaves RJ, Shepheard SL. Pathophysiology of migraine--new insights. Can J Neurol Sci 1999; 26 Suppl 3: S12-19.

[62] Buzzi M. Trigeminal pain pathway: Peripheral and central activation as experimental models of migraine. Func Neurol 2001; 16: 77-81.

[63] Holmlund A, Ekblom A, Hansson $\mathrm{P}$, et al. Concentration of neuropeptide substance $-\mathrm{p}$, neurokinin a, calcitonin gene related 
peptide, neuropeptide y and vasoactive intestinal polypeptide in the synovial fluid of the -human temporomandibular joint. Int J Oral Maxillofac Surg 1991; 20: 228-231

[64] Appelgren A, Appelgren B, Kopp S, et al. Relation between intraarticular temperature of the arthritic temporomandibular joint and presence of calcitonin gene-related peptide in the joint fluid. A clinical study. Acta Odontol Scand 1993; 51: 285-291.

[65] Zhang X, Chen Y, Wang C, et al. Neuronal somatic atp release triggers neuron-satellite glial cell communication in dorsal root ganglia. Proc Nat Acad Sci 2007; 104: 9864-9869.

[66] Neubert JK, Maidment NT, Matsuka Y, et al. Inflammationinduced changes in primary afferent-evoked release of substance $\mathrm{p}$ within trigeminal ganglia in vivo. Brain Res 2000; 871: 181-191.

[67] Li J, Vause C, Durham P. Calcitonin gene-related peptide stimulation of nitric oxide synthesis and release from trigeminal ganglion glial cells. Brain Res 2008; 1196: 22-32.

[68] Zhang Z, Winborn C, Marquez de Prado B, et al. Sensitization of calcitonin gene-related peptide receptors by receptor activitymodifying protein-1 in the trigeminal ganglion. J Neurosci 2007; 27: 2693-2703.

[69] Purkiss J, Welch M, Doward S, et al. Capsaicin-stimulated release of substance $\mathrm{p}$ from cultured dorsal root ganglion neurons: Involvement of two distinct mechanisms. Biochem Pharmacol 2000; 59: 1403-1406.

[70] Durham P, Cady R, Cady R. Regulation of calcitonin gene-related peptide secretion from trigeminal nerve cells by botulinum toxin type a: Implications for migraine therapy. Headache 2004; 44: 3542 .

[71] Rothwell N, Hopkins S. Cytokines and the nervous system ii: Actions and mechanisms of action. Trends Neurosci 1995; 18: 130136

[72] Vitkovic L, Bockaert J, Jacque C. "Inflammatory" Cytokines: Neuromodulators in normal brain? J Neurochem 2000; 74: 457471.

[73] Capuano A, De Corato A, Lisi L, et al. Proinflammatory-activated trigeminal satellite cells promote neuronal sensitization: Relevance for migraine pathology. Mol Pain 2009; 5: 43

[74] Fabbretti E, D'Arco M, Fabbro A, et al. Delayed upregulation of atp $\mathrm{p} 2 \mathrm{x}_{3}$ receptors of trigeminal sensory neurons by calcitonin gene-related peptide. J Neurosci 2006; 26: 6163-6171.

[75] Suadicani S, Cherkas P, Zuckerman J, et al. Bidirectional calcium signaling between satellite glial cells and neurons in cultured mouse trigeminal ganglia. Neuron Glia Biol 2009; 6: 1-9.

[76] Cady R, Schreiber C. Sinus headache or migraine? Considerations in making a differential diagnosis. Neurology 2002; 58: S10-14.

[77] Schreiber C, Hutchinson S, Webster C, et al. Prevalence of migraine in patients with a history of self-reported or physiciandiagnosed "Sinus" Headache. Arch Intern Med 2004; 164: 17691772.

[78] Cady R, Schreiber C, Farmer K. Understanding the patient with migraine: The evolution from episodic headache to chronic neurologic disease. A proposed classification of patients with headache. Headache 2004; 44: 426-435.

[79] Schreiber C, Hutchinson S, Webster C, et al. Prevalence of migraine in patients with a history of self-reported or physiciandiagnosed "Sinus" Headache. Arch Intern Med 2004; 164: 17691772.

[80] Ku M, Silverman B, Prifti N, et al. Prevalence of migraine headaches in patients with allergic rhinitis. Ann Allergy Asthma Immunol 2006; 97: 226-230.

[81] Damodaram S, Thalakoti S, Freeman SE, et al. Tonabersat inhibits trigeminal ganglion neuronal-satellite glial cell signaling. Headache 2009; 49: 5-20.

[82] Bensch G, Nelson H, Borish L. Evaluation of cytokines in nasal secretions after nasal antigen challenge: Lack of influence of antihistamines. Ann Allergy Asthma Immunol 2002; 88: 457-462.

[83] Repka-Ramirez S, Naranch K, Park Y, et al. Cytokines in nasal lavage fluids from acute sinusitis, allergic rhinitis, and chronic fatigue syndrome subjects. Allergy Asthma Proc 2002; 23: 185190.

[84] Bachert C, Wagenmann M, Hauser U. Proinflammatory cytokines: Measurement in nasal secretion and induction of adhesion receptor expression. Int Arch Allergy Immunol 1995; 107: 106-108.

[85] Bradding P, Mediwake R, Feather I, et al. Tnf alpha is localized to nasal mucosal mast cells and is released in acute allergic rhinitis. Clin Exp Allergy 1995; 25: 406-415.
[86] Pietrobon D. Migraine: New molecular mechanisms. Neuroscientist 2005; 11: 373-386.

[87] Morch C, Hu J, Arendt-Nielsen L, et al. Convergence of cutaneous, musculoskeletal, dural and visceral afferents onto nocicpetive neurons in the first cervical dorsal horn. Eur J Neurosci 2007; 26: 142-154.

[88] Piovesan E, Kowacs P, Oshinsky M. Convergence of cervical and trigeminal sensory afferents. Curr Pain Headache Rep 2003; 7 : 377-383.

[89] Freeman S, Patil V, Durham P. Nitric oxide-proton stimulation of trigeminal ganglion neurons increases mitogen-activated protein kinase and phosphatase expression in neurons and satellite glial cells. Neuroscience 2008; 157: 542-555.

[90] Arinci A, Ademoglu E, Aslan A, et al. Molecular correlates of temporomandibular joint disease. Oral Surg Oral Med Oral Pathol Oral Radiol Endod 2005; 99: 666-670.

[91] Takahashi T, Kondoh T, Ohtani M, et al. Association between arthroscopic diagnosis of temporomandibular joint osteoarthritis and synovial fluid nitric oxide levels. Oral Surg Oral Med Oral Pathol Oral Radiol Endod 1999; 88: 129-136.

[92] Ichikawa H, Sugimoto T. The co-expression of asic3 with calcitonin gene-related peptide and parvalbumin in the rat trigeminal ganglion. Brain Res 2002; 943: 287-291.

[93] Tanimoto T, Takeda M, Nasu M, et al. Immunohistochemical coexpression of carbonic anhydrase ii with kv1.4 and trpv1 in rat small-diameter trigeminal ganglion neurons. Brain Res 2005; 1044: 262-265.

[94] Bae Y, Oh J, Hwang S, et al. Expression of vanilloid receptor trpv1 in the rat trigeminal sensory nuclei. J Comp Neurol 2004; 478: 6271 .

[95] Diogenes A, Patwardhan A, Jeske N, et al. Prolactin modulates trpv1 in female rat trigeminal sensory neurons. J Neurosci 2006 26: 8126-8136

[96] Durham P, Russo A. Stimulation of the calcitonin gene-related peptide enhancer by mitogen-activated protein kinases and repression by an antimigraine drug in trigeminal ganglia neurons. J Neurosci 2003; 23: 807-815.

[97] Vause C, Durham P. Cgrp stimulation of inos and no release from trigeminal ganglion glial cells involves mitogen-activated protein kinase pathways. J Neurochem 2009; 110: 811-821.

[98] Kaminska B. Mapk signaling pathways as molecular targets for anti inflammatory therapy-from molecular mechanisms to therapeutic benefits. Biochem Biophsy Acta 2005; 1754: 253-262.

[99] Schindler J, Monahan J, Smith W. P38 pathway kinases as antiinflammatory drug targets. J Dent Res 2007; 86: 800-811

[100] Alstergren P. Cytokines in temporomandibular joint arthritis. Oral Dis 2000; 6: 331-334.

[101] Nishimura M, Segami N, Keneyama K, et al. Proinflammatory cytokines and arthroscopic findings of patients with internal derangement and osteoarthritis of temporomandibular joint. Br J Oral Maxillofac Surg 2002; 40: 68-71.

[102] Takeda M, Tanimoto T, Kadoi J, et al. Enhanced excitability of nociceptive trigeminal ganglion neurons by satellite glial cytokine following peripheral inflammation. Pain 2007; 129: 155-166.

[103] Wang X, Liu Y. Regulation of innate immune response by map kinase phosphatase-1. Cell Signal 2007; 19: 1372-1382.

[104] Garrett FG, Durham PL. Differential expression of connexins in trigeminal ganglion neurons and satellite glial cells in response to chronic or acute joint inflammation. Neuron Glia Biol 2009; 1-12.

[105] Suzuki I, Harada T, Asano M, et al. Phosphorylation of erk in trigeminal spinal nucleus neurons following passive jaw movement in rats with chronic temporomandibular joint inflammation. J Orofac Pain 2007; 21: 225-231.

[106] Thut P, Hermanstyne T, Flake N, et al. An operant conditioning model to assess changes in feeding behavior associated with temporomandibular joint inflammation in the rat. J Orofacial Pain 2007; 21: 7-18.

[107] Wang S, Lim G, Mao J, et al. Regulation of the trigeminal $\mathrm{nr} 1$ subunit expression induced by inflammation of the temporomandibular joint region in rats. Pain 2008; 10: 1-7.

[108] Xu Q, Garraway S, Weyerbacher A, et al. Activation of the neuronal extracellular signal-regulated kinase 2 in the spinal cord dorsal horn is required for complete freund's adjuvant-induced pain hypersensitivity. J Neurosci 2008; 28: 14087-14096. 
[109] Nakase T, Naus C. Gap junctions and neurological disorders of the central nervous system. Biochim Biophys Acta 2004; 1662: 149158.

[110] Schock S, Leblanc D, Hakim A, et al. Atp release by way of connexin 36 hemichannels mediates ischemic tolerance in vitro. Biochem Biophys Res Commun 2008; 368: 138-144.

[111] Toma I, Bansal E, Meer EJ, et al. Connexin 40 and atp-dependent intercellular calcium wave in renal glomerular endothelial cells. Am J Physiol Regul Integr Comp Physiol 2008; 294: R1769-1776.

[112] Cheriann P, Siller-Jackson A, Gu S, et al. Mechanical strain opens connexin 43 hemichannels in osteocytes: A novel mechanism for the release of prostaglandin. Mol Biol Cell 2005; 16: 3100-3106.

[113] Bruzzone S, Guida L, Zocchi E, Franco L, De Flora A. Connexin 43 hemi channels mediate ca2+-regulated transmembrane nad+ fluxes in intact cells. FASEB J 2001; 15: 10-12.
[114] Bennett M, Contreras JE, Bukauskas FF, Sáez JC. New roles for astrocytes: Gap junction hemichannels have something to communicate. Trends Neurosci 2003; 26: 610-617.

[115] Ebihara L. New roles for connexins. News Physiol Sci 2003; 18: 100-103.

[116] Ye Z, Wyeth MS, Baltan-Tekkok S, Ransom BR. Functional hemichannels in astrocytes: A novel mechanism of glutamate release. J Neurosci 2003; 23: 3588-3596.

[117] Hucho T, Levine JD. Signaling pathways in sensitization: Toward a nociceptor cell biology. Neuron 2007; 55: 365-376.

[118] Watkins L, Maier S. Beyond neurons: Evidence that immune and glial cells contribute to pathological pain states. Physiol Rev 2002; 82: 981-1011.

(C) Durham and Garrett; Licensee Bentham Open

This is an open access article licensed under the terms of the Creative Commons Attribution Non-Commercial License (http://creativecommons.org/licenses/by-nc/3.0/) which permits unrestricted, non-commercial use, distribution and reproduction in any medium, provided the work is properly cited. 